\title{
Transcriptome profiling of the response of Mycosphaerella graminicola isolates to an azole fungicide using cDNA microarrays
}

\author{
HANS J. COOLS*, BART A. FRAAIJE, TIM P. BEAN, JOHN ANTONIW AND JOHN A. LUCAS \\ Plant-Pathogen Interactions Division, Rothamsted Research, Harpenden, Hertfordshire AL5 2JQ, UK
}

\section{SUMMARY}

Resistance to azole antifungals is a major problem in the control of diseases caused by fungal pathogens of both humans and plants. Potential for the development of azole resistance in the wheat leaf blotch pathogen Mycosphaerella graminicola, the causal agent of the most economically significant foliar disease of wheat in north-western Europe, is now of particular concern after the recent emergence of widespread resistance to quinone outside inhibitor fungicides. Using a cDNA microarray representing around $25 \%$ of the genome, we have profiled the transcriptional response of $M$. graminicola to epoxiconazole, currently the most widely used azole fungicide on cereal crops. By comparing the transcription profiles of two M. graminicola isolates with contrasting sensitivities to epoxiconazole we show qualitative and quantitative differences in differentially expressed genes, including those involved in ergosterol biosynthesis, mitochondrial respiration and transport mechanisms. This represents the first study investigating the response of a plant pathogenic fungus to a fungicide using CDNA microarray technology.

\section{INTRODUCTION}

Septoria leaf blotch, caused by the ascomycete fungus Mycophaerella graminicola (anamorph: Septoria tritici) has been the most economically important fungal pathogen of winter wheat in the UK for over 20 years, causing estimated yield losses of $£ 30$ million per annum (Hardwick et al., 2001). None of the current commercially available wheat cultivars is fully resistant to the disease. Therefore, the programmed application of fungicides is the only effective control strategy. Groups of site-specific systemic fungicides including the methyl benzimidazole carbamates (MBCs), quinone outside inhibitors (Qols) and demethylation inhibitors (DMIs) have been used extensively against the disease since the early 1970s. However, the pathogen has quickly adapted and

*Correspondence: Tel.: +44 1582763 133; Fax: +44 1582760 981;

E-mail: hans.cools@bbsrc.ac.uk resistance to a number of chemical classes, including the MBCs and Qols, is now widespread in UK populations (Fraaije et al., 2003 , 2005). Resistance to the azole group of DMI fungicides has not yet emerged. However, owing to the current lack of alternative chemistry to control the disease, and the consequent increased reliance on azoles, there is concern that resistance to this group could develop, particularly in light of the recent decline in efficacy of a range of azoles in controlling Septoria leaf blotch (Clark, 2006).

Azole resistance in plant pathogenic fungi has previously developed slowly and loss of efficacy in practice remains rare. By contrast, resistance to azoles in human pathogens is a major problem, particularly in immunosuppressed patients in whom control of recurrent infections caused by opportunistic pathogens, for example Candida albicans, is commonly compromised by resistance. The adaptation of human fungal pathogens to azoles demonstrates the biological potential for resistance development, thereby providing a paradigm for the evolution to resistance in other pathogens, including those of plants. Consequently, studies of the mechanisms underlying changes in azole sensitivity in plant pathogens have to date focused on those well-characterized in human pathogens, principally $C$. albicans.

The most frequently identified azole resistance mechanism in C. albicans is up-regulation of active efflux proteins, ATP-binding cassette $(A B C)$ transporters and major facilitators (MFs), which reduce the intracellular concentration of the fungicide (Perea et al., 2001). Active efflux has also been shown to contribute to azole resistance in plant pathogens, although identified most frequently in laboratory-generated mutants. Such laboratory mutants of $M$. graminicola resistant to azoles with altered expression of $A B C$ transporter genes have been shown to revert to an azolesensitive phenotype when transporter genes are disrupted (Zwiers et al., 2002). Efflux pump over-expression has only been shown to confer directly azole resistance in field isolates of Penicillium italicum (Nakuane et al., 1998). Studies of M. graminicola field isolates collected in the 1990s showed differences in both basal and induced levels of $A B C$ transporter gene transcript, although no correlation between increased expression and azole sensitivity was evident (Stergiopoulos et al., 2003). Similarly, studies of more recent $M$. graminicola field isolates from the UK 
failed to establish any direct relationship between over-expression and decreased azole sensitivity (Cools et al., 2005a). Therefore, the contribution of active efflux to azole resistance in field populations of plant pathogens, although implicated, remains unclear.

The most common mechanism of resistance to site-specific fungicides is modification of the target protein. The target for azole fungicides is the cytochrome P450 sterol $14 \alpha$-demethylase (CYP51), an essential enzyme of the ergosterol biosynthesis pathway, the predominant sterol in the membranes of fungi. Alterations in CYP51 have been associated with changes in azole sensitivity in fungal pathogens of humans and plants, although the impacts of these changes are best characterized in the human pathogen C. albicans with a number of CYP51 mutations functionally characterized (Kelly et al., 1999; Lamb et al., 2000; Sanglard et al., 1998). Mutations in CYP51 encoding changes at residues equivalent to those known to affect an azole resistant phenotype in C. albicans have also been identified in plant pathogens, most notably the equivalent to substitution $\mathrm{Y} 132 \mathrm{~F} / \mathrm{H}$ in powdery mildews (Delye et al., 1997, 1998; Wyand and Brown, 2005). This substitution has also been identified in $M$. graminicola (Y137F) (Cools et al., 2005b). Our studies have also identified CYP51 alterations not previously associated with azole resistance in human or plant pathogenic fungi. Substitution $1381 \mathrm{~V}$, for example, appears to be differentially selected by members of the azole class of fungicides (Fraaije et al., 2007). In C. albicans, combinations of CYP51 mutations confer greater reductions in azole sensitivity than single mutations. In plant pathogens mutations also appear to be accumulating, for example in Blumeria graminis f. sp. hordei (Wyand and Brown, 2005) and M. graminicola (Cools et al., 2005b; Fraaije et al., 2007), although as isolates with identical CYP51 mutations can have different azole sensitivities, the exact impact of target site changes on azole efficacy in the field remains unknown (Cools et al., 2006).

In this study, we have used cDNA microarrays to profile the transcriptional response of $M$. graminicola to epoxiconazole, currently the azole most widely used to control this disease. We have compared the expression profiles of an azole-sensitive and less sensitive $M$. gramincola isolate to a single discriminatory dose to determine whether decreased azole sensitivity is conferred by mechanisms other than mutations in the CYP51 gene. To our knowledge, this is the first investigation into the response of a plant pathogenic fungus to a fungicide using microarrays. We describe the effect of epoxiconazole treatment on genes involved in ergosterol biosynthesis, mitochondrial respiration and toxicant transport. Furthermore, we report both qualitative and quantitative differences in basal and induced gene expression between M. graminicola isolates with different CYP51 mutations and contrasting sensitivities to azoles.

\section{RESULTS}

\section{Characterization of isolates}

The calculated $\mathrm{EC}_{50}$ to epoxiconazole for isolate $\mathrm{G} 303$ is around 25-fold higher than sensitive isolate IP0323. CYP51 genes of IP0323 and G303 have been previously sequenced (Cools et al., 2005b), with a number of encoded amino acid changes identified in G303. Some of these alterations have been correlated with reduced azole sensitivity (Cools et al., 2005a,b; Fraaije et al., 2007). $\mathrm{EC}_{50}$ values for Rothamsted, Hertfordshire, isolates obtained in 2006 ranged from $0.164 \mathrm{mg} / \mathrm{L}$ (isolate R6-40) to $2.19 \mathrm{mg} / \mathrm{L}$ (R6-31). Multiple CYP51 mutations were identified in all isolates (Table 1). All isolates grew at similar rates in vitro (data not shown).

\section{Constitutive differences in gene expression between IP0323 and G303}

Ten genes were more highly expressed, beyond a two-fold cut off, in less sensitive isolate G303 compared with sensitive isolate IP0323 (Table 2). These include those encoding a drug transporter (unisequence Id: mga1012f) protein, a cell surface glycoprotein (mg[0175]), thought to be a flocculin, stress response protein rds1 (mg[0194]), a myo-inositol transport protein (mgb17a02f), a mitochondrial transport protein (mgb12c11f), and an unknown gene encoding a homologue of the antibiotic response protein CipA of Aspergillus nidulans (mgc03e06f).

Table 1 M. graminicola isolates used in this study.

\begin{tabular}{lllll}
\hline Isolate & Year & Location & $\begin{array}{l}\text { Epoxiconazole } \\
\mathrm{EC}_{50}(\mathrm{mg} / \mathrm{L})\end{array}$ & CYP51 alteration(s) \\
\hline IP0323 & 1981 & Netherlands & 0.06 & none \\
G303 & 2003 & Kent & 1.47 & L50S, D138G, S188N, A379G, I381V, DY459-G460, N513K \\
R6-31 & 2006 & Hertfordshire & 2.19 & Y137F \\
R6-32 & 2006 & Hertfordshire & 0.698 & L50S, S188N, I381V, DY459-G460, N513K \\
R6-40 & 2006 & Hertfordshire & 0.164 & L50S, I381V, Y459D \\
R6-55 & 2006 & Hertfordshire & 0.976 & L50S, I381V, Y461H \\
\hline
\end{tabular}


Table 2 Genes differentially expressed in isolate G303 compared with isolate IP0323 in the absence of epoxiconazole.

\begin{tabular}{|c|c|c|c|c|}
\hline Functional category (MIPS) & EST ID & $\begin{array}{l}\text { Unisequence } \\
I^{*}\end{array}$ & Best match; accession no. & $\begin{array}{l}\text { Mean fold } \\
\text { change in } \\
\text { expression }\end{array}$ \\
\hline cell wall biogenesis & mga0371 & mga0371 & $N$-acetyl- $\beta$-D-glucosaminidase (exochitinase); AAL78815 & +2.1 \\
\hline drug transporters & mga1012f & mga1012f & multidrug resistance protein; EAA28910 & +3.4 \\
\hline electron transport proteins & mga0964 & mga0964 & subunit IV of cytochrome c oxidase; EAA33815 & +2.1 \\
\hline mitochondrial transport & mgb12c11f & mgb12c11f & $\begin{array}{l}\text { protein of the inner mitochondrial membrane, required } \\
\text { for import of mitochondrial matrix proteins; EAA36389 }\end{array}$ & +2.4 \\
\hline \multirow[t]{2}{*}{ organization of cell wall } & mga0959f & $\mathrm{mg}[0175]$ & cell surface flocculin (glycoprotein); T45462 & +3.4 \\
\hline & $\mathrm{mgc02g01f}$ & $\mathrm{mg}[0558]$ & $\begin{array}{l}\text { homologue of clock controlled protein } 6 \\
\text { (N. crassa) (cell surface glycoprotein); Q01302 }\end{array}$ & +2.1 \\
\hline protein modification & $\mathrm{mg} 12 \mathrm{~h} 01 \mathrm{f}$ & $\mathrm{mg} 12 \mathrm{~h} 01 \mathrm{f}$ & nuclear protein arginine methyltransferase; EAA34674 & +2.1 \\
\hline $\begin{array}{l}\text { sugar, carbohydrate and } \\
\text { metabolite transporters }\end{array}$ & mgb17a02f & mgb17a02f & myo-inositol transport protein; EAA28903 & +2.6 \\
\hline stress response & mga0334 & $\mathrm{mg}[0194]$ & stress response protein (rds1); CAD21425 & +3.2 \\
\hline unclassified protein & $\mathrm{mgc03e} 06 f$ & $\mathrm{mg}[1000]$ & $\begin{array}{l}\text { homologue of CipA (E. nidulans), associated } \\
\text { with antibiotic response; CAC87270 }\end{array}$ & +2.8 \\
\hline
\end{tabular}

*Sequences available at http://cogeme.ex.ac.uk/

Table 3 Genes differentially expressed in isolate IP0323 after $24 \mathrm{~h}$ exposure to $2 \mathrm{mg} / \mathrm{L}$ epoxiconazole.

\begin{tabular}{lllll}
\hline $\begin{array}{l}\text { Functional category } \\
\text { (MIPS) }\end{array}$ & EST ID & Unisequence ID* & Best match; accession no. & Mean fold change \\
\hline $\begin{array}{l}\text { tetracyclic and pentacyclic } \\
\text { triterpene (steroids and } \\
\text { hopanoids) biosynthesis }\end{array}$ & mgc01h02f & mg[0887] & C-4 sterol methyl oxidase; EAA33004 & +4.2 \\
& & & & \\
& $\begin{array}{l}\text { mga0888f } \\
\text { mgc01f02f }\end{array}$ & $\begin{array}{l}\text { mga0888f } \\
\text { mg[0869] }\end{array}$ & $\begin{array}{l}\text { C14-sterol reductase; 013597 } \\
\text { sterol C-22 desaturase; BAC01140 }\end{array}$ & +7.7 \\
\hline
\end{tabular}

*Sequences available at http://cogeme.ex.ac.uk/

\section{Gene expression in response to epoxiconazole treatment}

Genes up-regulated in response to $24 \mathrm{~h}$ exposure to a lethal (Table 1) dose of epoxiconazole in isolate IP0323, beyond a twofold cut-off, all encoded components of the sterol biosynthesis pathway: C-4 sterol methyl oxidase (erg25, mg[0887]), C14-sterol reductase (erg24, mga0888f) and C-22 sterol desaturase (erg5, $\mathrm{mg}$ [0869]) (Table 3). Expression of erg5, erg24 and erg25 in isolate $\mathrm{G} 303$ also increased after treatment with a non-lethal (Table 1) dose of epoxiconazole, although transcript levels were lower than those detected for IP0323 (Table 4). Furthermore, mga1391f encoding hydroxymethylglutaryl-CoA synthase (HMGCoA synthase, erg13), an enzyme involved in mevalonate synthesis, a precursor of ergosterol biosynthesis, is up-regulated in G303. The orthologous gene is similarly responsive in C. albicans (De Backer et al., 2001). Genes other than those encoding proteins involved in sterol biosynthesis up-regulated in G303 include a putative hexose transporter, $\mathrm{mg}$ [0271], a ubiquinol cytochrome $\mathrm{c}$ reductase subunit, $\mathrm{mg}[1446]$, and an unknown protein homologous to FA01 of Cryptococcus neoformans, mgc03g08f.

\section{Hierarchical clustering of genes involved in sterol biosynthesis}

Hierarchical clustering of genes in the same functional category (MIPS) associated genes by relative expression profile. This enabled genes that were not differentially expressed beyond the stringent two-fold cut-off to be analysed. Cluster analysis confirmed sterol biosynthesis genes erg25 (mg[0887]), erg24 (mga0888f) and erg5 (mg[0869]) as up-regulated in response to $24 \mathrm{~h}$ epoxiconazole treatment (Fig. $1 \mathrm{~A}$ ). In addition, expression of genes encoding a sterol isomerase (mga1231f) and the sterol C-24 reductase (mgc19a12f) were identified by clustering as azole induced. Expression of mgc19a12f, encoding erg4, the sterol C-24 reductase, is only induced in $\mathrm{G} 303$, perhaps reflecting continued ergosterol production in this isolate. Genes originally annotated as involved in sterol biosynthesis that are unaffected or down-regulated by azole treatment include mg[1270] encoding a farnesyl diphosphate synthetase (erg20), mg[0650] suggested to encode the sterol C-24 methyltransferase (erg6) and other sterolassociated proteins, for example mg1265f encoding a hydroxysteroid dehydrogenase. However, Blastx searches fail to identify 
A

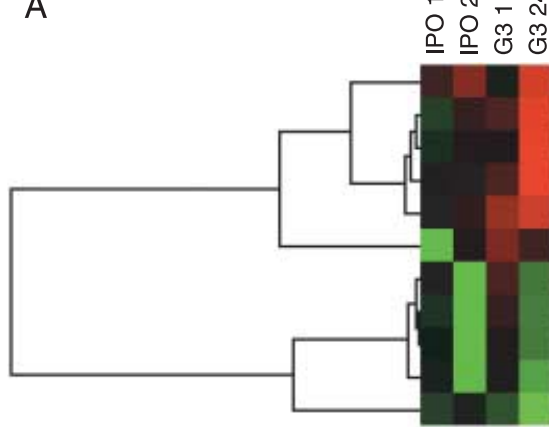

mgal265f mga0406f mgal631f mgb0319f mgb 16 g0 $2 f$ mgb0245f mga0888f mgal231f mgc01h02f mgc0lf02f mgcl9al2f

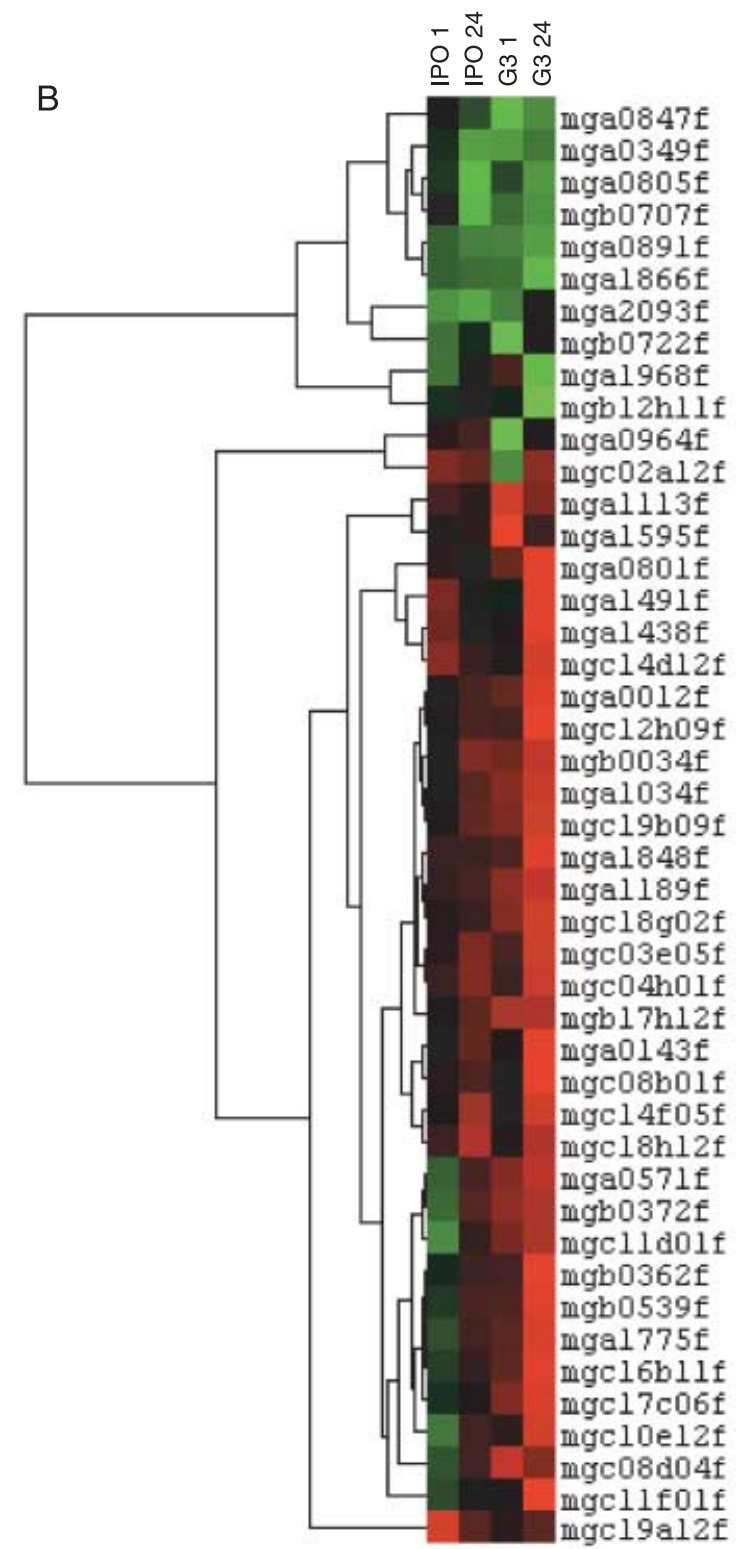



(mg [0372])

(mg[0201])

(mg[0306])

( $\operatorname{mg}[1446])$

(mg [0432])

( $\mathrm{mg}[0432])$

(ng $[0356])$

( $\operatorname{mg}[0489]$ )

( $\operatorname{mg}[1340]$ )

( $\operatorname{sig}[0011]$ )

( $\operatorname{mg}[0417])$

(mg[0417])

(mg $[1026])$

(mg[0432])

( $\mathrm{mg}[0417]$ )

(mg[0011])

( $\mathrm{mg}[0283]$ )

(mg [0635])

(mg[0549])

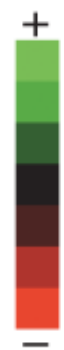

Fig. 1 Hierarchical clustering showing the patterns of expression of genes involved in sterol biosynthesis (A) and electron transport (B) in isolate IP0323 after $1 \mathrm{~h}$ (IPO 1) and $24 \mathrm{~h}$ (IPO 24) and G303 after $1 \mathrm{~h}$ (G3 1) and $24 \mathrm{~h}$ (G3 24) of epoxiconazole treatment. Colour bars display normalized $\log _{2}$ (green/red) ratio, with untreated IP0323 as the reference point. EST IDs are shown. Unisequence IDs are given in parentheses. 
Table 4 Genes differentially regulated in isolate G303 after $24 \mathrm{~h}$ exposure to $2 \mathrm{mg} / \mathrm{L}$ epoxiconazole.

\begin{tabular}{|c|c|c|c|c|}
\hline Functional category (MIPS) & EST ID & $\begin{array}{l}\text { Unisequence } \\
I^{*}\end{array}$ & Best match; accession no. & $\begin{array}{l}\text { Mean fold } \\
\text { change in } \\
\text { expression }\end{array}$ \\
\hline electron transport proteins & mgb12h11f & $\mathrm{mg}[1446]$ & ubiquinol cytochrome-c reductase subunit; P48503 & +2.1 \\
\hline isoprenoid biosynthesis & mga1391f & mga1391f & $\begin{array}{l}\text { hydroxymethylglutaryl-CoA synthase, } \\
\text { functions in mevalonate synthesis; T49718 }\end{array}$ & +2.2 \\
\hline \multirow{4}{*}{$\begin{array}{l}\text { sugar, carbohydrate and metabolite transporters } \\
\text { tetracyclic and pentacyclic triterpene } \\
\text { (steroids and hopanoids) biosynthesis }\end{array}$} & mgb0930f & $\mathrm{mg}[0271]$ & hexose transporter; EAA28833 & +2.1 \\
\hline & mgc01h02f & $\mathrm{mg}[0887]$ & C-4 sterol methyl oxidase; EAA33004 & +2.9 \\
\hline & mga0888f & mga0888f & C14-sterol reductase; 013597 & +3.6 \\
\hline & $\operatorname{mgc01f02f~}$ & $\mathrm{mg}[0869]$ & sterol C-22 desaturase; BAC01140 & +4.5 \\
\hline unclassified protein & mgc03g08f & $\operatorname{mgc03g08f}$ & homologue of FAO1 (Cryptococcus neoformans); AAN75712 & +2.8 \\
\hline
\end{tabular}

*Sequences available at http://cogeme.ex.ac.uk/

most of these genes (mga1265, mg[0216], mgb16g02f and mgb0245f) as sterol-associated. The exceptions are mg[1270], which has homology to erg20 of Gibberella zeae (2e-50), and mg[0650], homologous to erg6 of Neurospora crassa (1e-63).

\section{Hierarchical clustering of genes encoding components of the mitochondrial electron transport chain}

Clustering of genes encoding components of the mitochondrial electron transport chain revealed a number up-regulated in response to epoxiconazole (on average around 1.5 -fold), particularly in isolate IP0323 (Fig. 1B). Interestingly, most azoleresponsive genes are mitochondrially encoded. For example, subunits 1-7 of the NADH-ubiquinone oxidoreductase complex are mitochondrially encoded in fungi and higher eukaryotes. All of these represented on the microarray (subunits $1(\mathrm{mg}[0372]), 2$ (mg[0201]; mga0891f), 4 (mga1866f; mga2093f) and 5 (mga0805f)) are up-regulated in response to epoxiconazole treatment. The remaining $\mathrm{NADH}$-ubiquinone oxidoreductase subunits are nuclear encoded. These include the 51-kDa (mg[1340]), 78-kDa (mga[0489]) and 20.8-kDa (mgc02a12f) subunits, all either unaffected or down-regulated in this study. Similarly, cytochrome $b$ (mgb0707f) and subunit III of the cytochrome c oxidase (mg[0306]) are mitochondrially encoded, with smaller subunits (e.g. subunit $V$ (mgc14d12f), VI (mga1848f)) encoded in the nucleus.

\section{Validation of microarray data by quantitative RT-PCR and analysis of Rothamsted 2006 isolates}

Genes selected for analysis by quantitative RT-PCR were those either constitutively up-regulated in isolate G303 (mga1012f (Fig. 2A) and mg[0194] (Fig. 2B)), involved in sterol biosynthesis (mga0888f (Fig. 2C), and the CYP51/erg11 azole target (Fig. 2D), absent from the microarray) and responsive to epoxiconazole, but not directly involved in sterol biosynthesis (mg[0271]
(Fig. 2E) and mgc03g08f (Fig. 2F)). In general, quantitative RTPCR results correlated well with microarray data, although for some genes, particularly mga0888f, fold changes in expression were greater, perhaps reflecting the greater dynamic range of real-time PCR.

Analysis of the expression of mg1012f (Fig. 3A) and epoxiconazole-responsive genes mg[0271] (Fig. 3B) and mgc03g08f (Fig. 3C) in M. graminicola isolates with varying azole sensitivities obtained from an untreated field at Rothamsted in 2006 revealed differences in constitutive and azole-induced transcript levels between isolates. For example, a high basal level of mga1012f expression was only detected in R6-55, with expression slightly reduced after epoxiconazole treatment, similar to results for $\mathrm{G} 303$. In the remaining isolates mga1012f expression was generally unaffected by azole. Expression of $\mathrm{mg}[0271]$ was induced by epoxiconazole treatment in all isolates; however, transcript levels varied between isolates with highest basal (6.2-fold) and induced (9.8-fold) expression levels detected for the least sensitive isolate R6-31. Mean expression levels of mgc03g08f, the predicted iron/ascorbate oxidoreductase gene, varied greatly between isolates. A 53-fold increase (relative to the calibrator sample) in transcript level was detected after epoxiconazole treatment in isolate R6-55. In no other isolates was mgc03g08f expression induced to the same extent.

\section{DISCUSSION}

Microarray transcriptional profiling of the response of human fungal pathogens to antifungals has already proved successful in identifying novel genes and/or gene families expressed upon exposure to these compounds and involved in the acquisition of resistance (De Backer et al., 2001; Ferreira et al., 2006; Rogers and Barker, 2003). This is the first study using microarray technology to profile the response of a plant pathogen when exposed to a fungicide. By analysing the expression of expressed sequence 

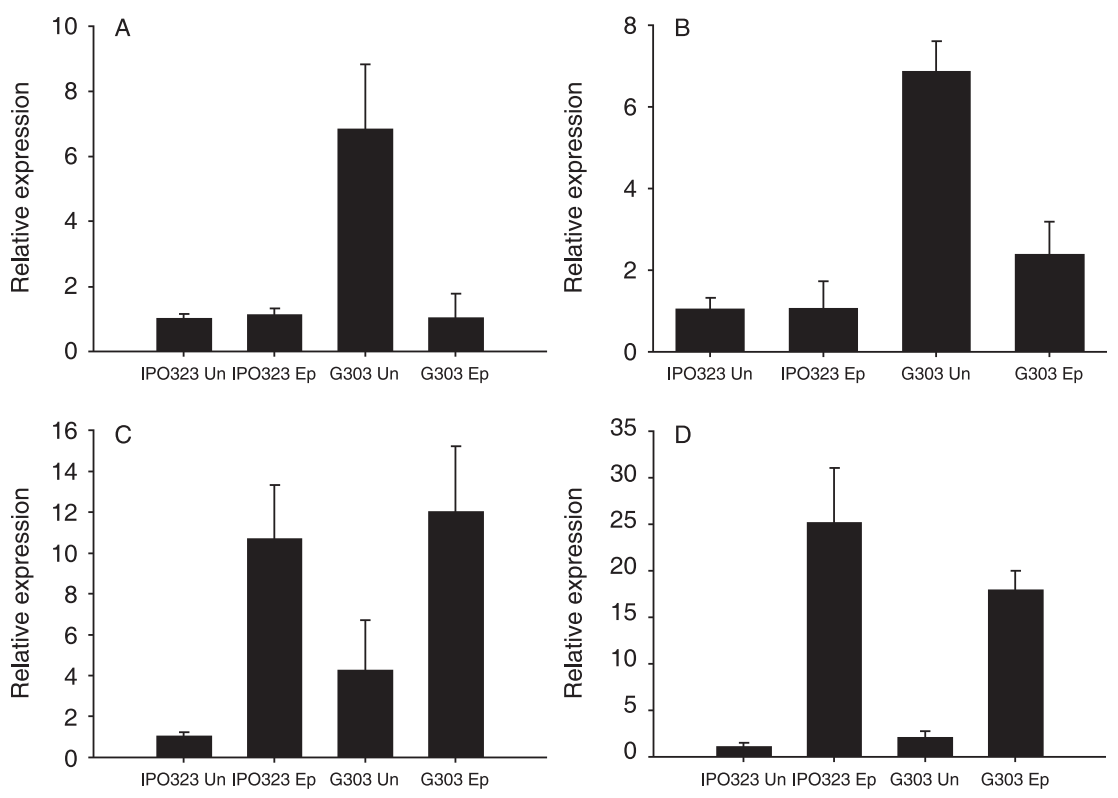

Fig. 2 Validation of microarray results using quantitative RT-PCR for genes identified as differentially expressed in microarray experiments.
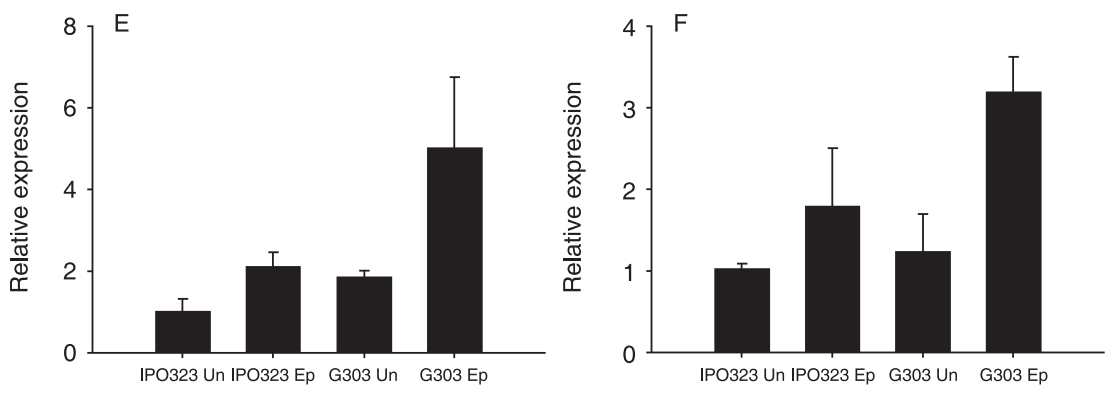
Shown are relative expression values in untreated IP0323 (IP0323 Un) and after $24 \mathrm{~h}$ of epoxiconazole treatment (IP0323 Ep) and untreated G303 (G303 Un) and after $24 \mathrm{~h}$ of epoxiconazole treatment (G303 Ep). Unisequences IDs of genes analysed are mga1012f(A), mg[0194] (B), CYP51/erg11 (C, not represented on the microarray), mga0888f (D), mg[0271] (E) and mg03g08f (F). Mean of two replicate experiments with standard deviations are shown.

tags (ESTs), predicted to cover a quarter of the genome of the wheat leaf blotch pathogen M. graminicola, upon exposure to the azole fungicide epoxiconazole, we have confirmed the effect of this compound on ergosterol biosynthesis, and, in addition, demonstrated an impact on the expression of components of the respiratory chain, predominantly those encoded in the mitochondrial genome. Comparison of the expression profile of an isolate less sensitive to azole (G303), carrying mutations in the targetencoding CYP51 gene, with the sensitive isolate (IP0323), both in the absence and in the presence of epoxiconazole, has identified differentially expressed genes that potentially have a role in reducing azole sensitivity in G303. Subsequent quantitative RTPCR analyses of the expression of these candidate genes in four more recently collected isolates with varying levels of azole sensitivity suggest these genes may also contribute to a less sensitive phenotype in other M. graminicola isolates.

\section{Response of genes involved in ergosterol biosynthesis}

Genes encoding components of the ergosterol biosynthesis pathway that are represented on the microarray were consistently the most responsive to treatment, confirming that this pathway is the primary target for epoxiconazole and responds to changes in ergosterol levels. Most ergosterol biosynthesis genes were upregulated in both isolates. The exceptions were erg4 (mgc19a12f), encoding the sterol $\mathrm{C}-24$ reductase, which was only responsive in isolate $\mathrm{G} 303$, erg20 ( $\mathrm{mg}[1270]$ ) encoding a farnesyl diphosphate synthetase, and erg6 (mg[0650]), encoding the sterol C-24 methyltransferase. Expression of both erg20 and erg6 was unaffected by azole treatment in isolate IP0323 and decreased in G303.

The sterol C-24 reductase catalyses the final step in ergosterol biosynthesis. Therefore, differential expression of erg 4 between isolates in the presence of epoxiconazole may reflect differences in the capacity of isolates to complete ergosterol biosynthesis. Consistent with this study, expression of erg20 is not affected by azole treatment in Saccharomyces cerevisiae (Bammert \& Fostel 2000) and C. albicans (De Backer et al., 2001). The observed effect of epoxiconazole treatment on erg6 expression, however, conflicts with previous studies of both $S$. cerevisiae (Agarwal et al., 2003; Bammert \& Fostel, 2000) and C. albicans (De Backer et al., 2001). This discrepancy with earlier studies and other data presented here, particularly the up-regulation of erg5, erg24 and 

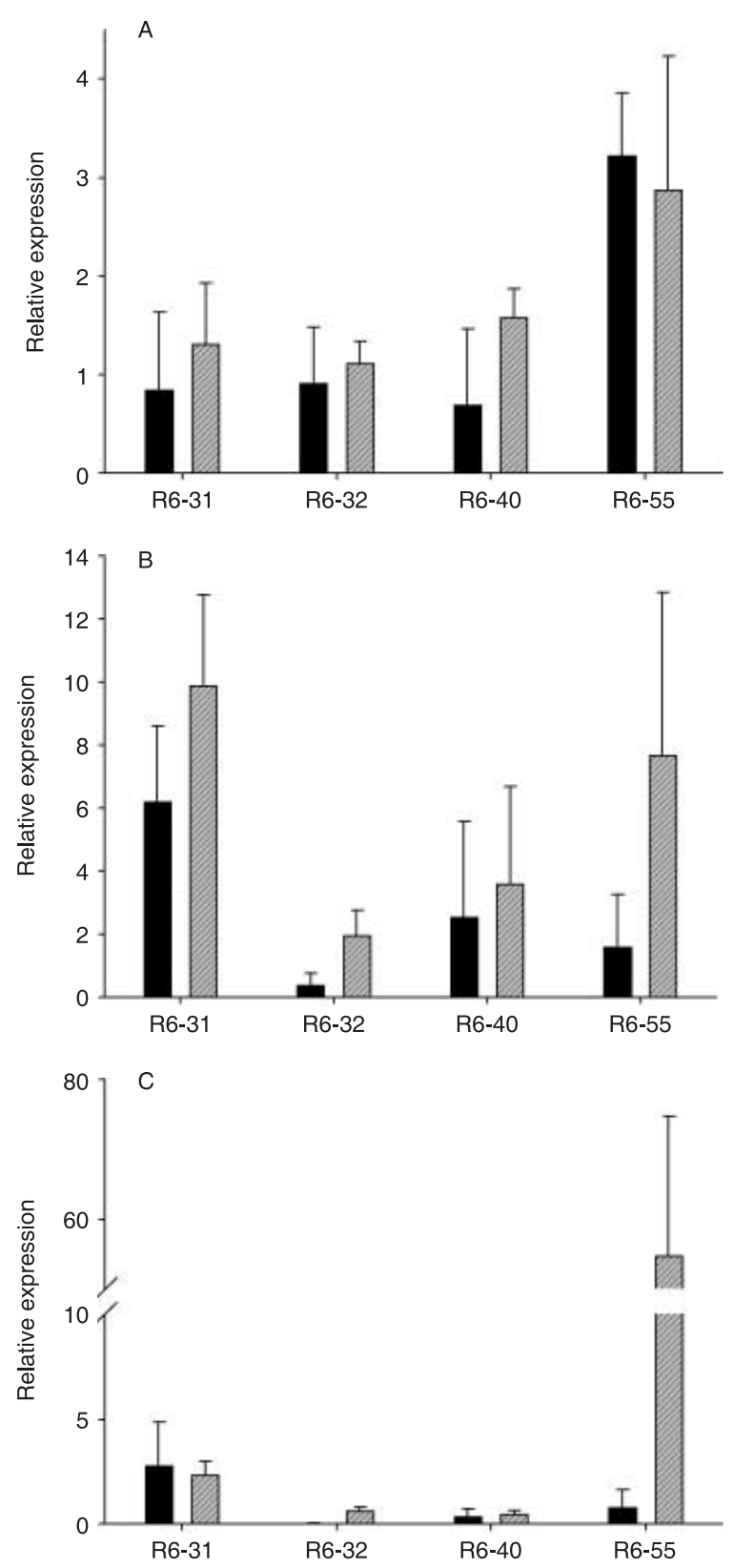

Fig. 3 Quantitative RT-PCR analysis of the expression of mga1012f (A), $\mathrm{mg}[0271]$ (B) and mgc03g08f (C) in isolates obtained from Rothamsted, Hertfordshire, in 2006 (R6-31, R6-32, R6-40 and R6-55). Expression analyses were carried out on untreated (filled) and $24 \mathrm{~h}$ epoxiconazole-treated (shaded) samples using untreated IP0323 as the reference. Mean of two replicate experiments and standard deviations are shown. For clarity, a break (10-50) has been inserted into the $y$-axis of graph C.

erg25 in response to azole, suggests this gene may not be correctly annotated and requires further analysis to establish its precise function. In Aspergillus fumigatus, the only filamentous fungus to be analysed after antifungal treatment by microarray thus far, expression of erg24 and erg25 also increased upon expo- sure to voriconazole (Ferreira et al., 2006), although unlike this (as shown by quantitative RT-PCR) and other studies (Agarwal et al., 2003; De Backer et al., 2001; Liu et al., 2005) expression of both CYP51/erg11 paralogues in A. fumigatus decreased upon treatment. Amongst the remaining genes predicted to encode components of the ergosterol biosyntheisis pathway, EST mga1231f, suggested to encode a sterol isomerase, is up-regulated upon azole exposure. Although homology searches suggest this gene does not encode erg2, evidence here does suggest mga1231f responds to changes in ergosterol levels.

The mechanisms responsible for erg gene up-regulation upon azole exposure remain unclear. However, consistent with other studies of S. cerevisiae (Agarwal et al., 2003; Bammert and Fostel, 2000) and C. albicans (De Backer et al., 2001; Liu et al., 2005), greatest changes in gene expression levels in M. graminicola after azole treatment were detected for genes encoding components functioning downstream of CYP51/erg11 (Fig. 4). This suggests that erg gene expression is induced specifically by ergosterol depletion, as has previously been proposed (Agarwal et al., 2003; De Backer et al., 2001; Liu et al., 2005). Alteration of erg gene expression, particularly up-regulation of erg11/CYP51, has been shown to confer azole resistance both in human (Rogers and Barker, 2003; White, 1997) and in plant pathogenic fungi (Hamamoto et al., 2000; Schnabel and Jones, 2001). In this study, no constitutive differences in erg gene expression were evident in microarray studies when the less sensitive isolate $\mathrm{G} 303$ was compared with the sensitive isolate IP0323. After $24 \mathrm{~h}$ exposure to azole, levels of erg gene up-regulation were similar between isolates. Quantitative RT-PCR analysis did suggest some differences in basal erg11/CYP51 and erg24 transcript levels. For example, erg11/CYP51 expression appeared around two-fold higher in G303. However, in the absence of corroborative microarray data, it is difficult to conclude an impact of altered erg gene expression on reducing azole sensitivity in G303.

\section{Response of genes involved in mitochondrial electron transport}

The relationship between ergosterol biosynthesis and mitochondrial respiration has long been established. For example, certain steps of ergosterol biosynthesis are dependent on mitochondrial development in yeast (Daum et al., 1998). Yet the effect of azoles on mitochondrial function is not clearly defined. In this study, hierarchical clustering revealed that a number of genes encoding components of the mitochondrial electron transport chain were up-regulated (between 1.5- and 1.8-fold) by epoxiconazole treatment. Microarray and erg mutant studies of $S$. cerevisiae (Bammert and Fostel, 2000) also demonstrated increased transcript levels of respiratory chain components after azole treatment, including members of the cytochrome c oxidase and cytochrome $\mathrm{c}$ reductase complexes and subunits of ATP synthase. Unlike the 


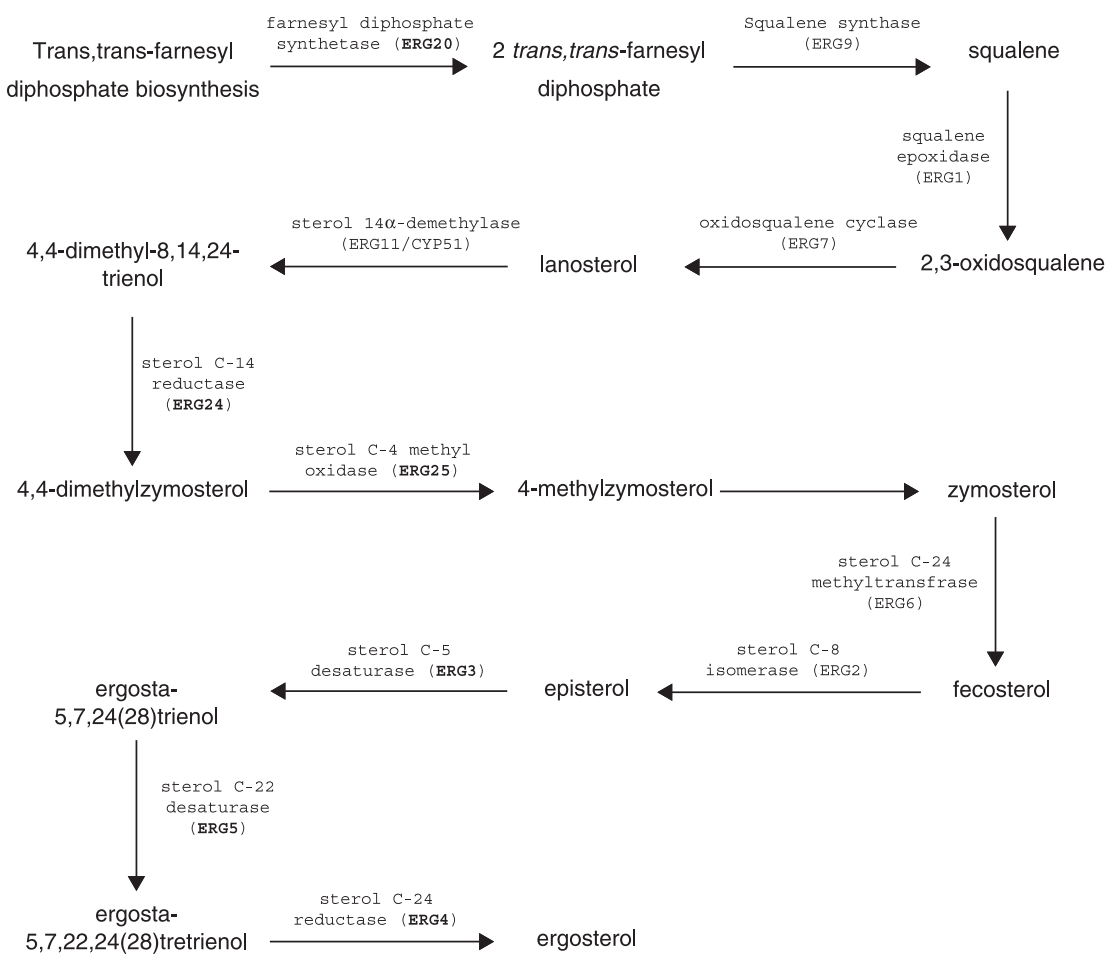

Fig. 4 Schematic of the ergosterol biosynthesis pathway from farnesyl pyrophosphate. Genes represented on the microarray are shown in bold type. findings presented here, however, Bammert and Fostel (2000) also observed increased transcript levels of known oxidative stress response genes, leading to the suggestion that perturbation of ergosterol biosynthesis by mutation or azole, and the consequent accumulation of sterol precursors, indirectly affects mitochondrial electron transport, leading to the generation of reactive oxygen species (ROS). Other microarray studies of fungi including $C$. albicans and $A$. fumigatus have not observed changes in electron transport gene expression upon azole exposure; however, studies of the transcriptional response of the bacterial pathogen Mycobacterium tuberculosis to azole treatment did identify responsive components of the respiratory chain (Boshoff et al., 2004).

Studies of petite mutants of $S$. cerevisiae and Candida glabrata provide additional evidence for an interaction between azole and mitochondria. In both S. cerevisiae (Kenna et al., 1989) and C. glabrata (Sanglard et al., 2001) azoles have been shown to be potent inducers of petite mutagenesis and, furthermore, petite mutants are highly resistant to azoles (Brun et al., 2004; Sanglard et al., 2001). Brun et al. (2004) suggested that azoles induce petite mutagenesis by directly inhibiting mitochondrial function, causing an accumulation of ROS and leading to mitochondrial damage. A direct interaction of azoles with components of the respiratory chain has previously been demonstrated using isolated mitochondria from C. albicans (Shigematsu et al., 1982).

Interestingly, most of the components of the respiratory chain which were up-regulated in response to epoxiconazole treatment in this study are mitochondrially encoded. The exceptions are the nuclear encoded NADH-ubiquinone oxidoreductase 49-kDa (mga1968) subunit and cytochrome $\mathrm{c}$ reductase subunit VII ( $\mathrm{mg}[1446])$, both of which are only responsive in isolate G303. Therefore, consistent with previous studies, epoxiconazole treatment of $M$. graminicola induces the expression of genes involved in mitochondrial respiration (Bammert and Fostel, 2000; Boshoff et al., 2004); however, it remains to be investigated whether this induction is specific to mitochondrially encoded components of the respiratory chain.

\section{Response of genes encoding transporter proteins}

In this study, only two genes encoding transport proteins were differentially expressed between isolates, or induced upon epoxiconazole treatment. mga1012f, encoding a vacuolar glutathione $S$-conjugate $A B C$ transporter, potentially involved in drug resistance, is constitutively over-expressed in isolate G303. Upon epoxiconazole exposure, however, expression of this gene decreases in this isolate, suggesting this gene is not contributing to reducing azole sensitivity in G303. Homology searches against the fungal genome database identify mga1012f as a vacuolar glutathione $S$-conjugate $A B C$ transporter. These proteins are members of the multidrug resistance-related family of $A B C$ transporters, primarily involved in the detoxification of metals (De Waard et al., 2006). No transporters of this type have previously been associated with altered azole sensitivity, although 
coexpression of an $A B C$ transporter and a glutathione-Stransferase gene has been suggested to be involved in voriconazole detoxification in A. fumigatus (Ferreira et al., 2006).

Microarray analysis demonstrated increased expression of $\mathrm{mg}$ [0271], encoding a putative hexose transporter, upon epoxiconazole treatment in G303. Expression of this gene was also shown subsequently by quantitative RT-PCR to be induced in isolate IP0323, although not to the same extent (around twofold in IP0323 compared with six-fold in G303). Expression of this gene in four more recently obtained $M$. graminicola isolates appears to be similarly affected by azole treatment. Interestingly, the highest constitutive and induced transcript levels of mg[0271] were detected in the least sensitive isolate R6-31. Hexose transporters of the major facilitator superfamily have been previously implicated in resistance to drugs. D-Hexose transporters have been shown to modulate drug accumulation in Xenopus oocytes expressing murine $M d r 1 b$, an $A B C$ transporter that confers a multidrug-resistant phenotype (Vera et al., 1991). HXT9 and HXT11, two $S$. cerevisiae hexose transporter genes that have homology to $\mathrm{mg}[0271]$ (5e-12), have also been implicated in a multidrugresistant phenotype (Nourani et al., 1997). Both HXT9 and HXT11 are Pdr1p and Pdr3p targets, transcriptional regulators of $A B C$ transporters required for drug resistance in yeast. Interestingly, although HXT9 and HXT11 are co-regulated with multidrug transporter genes, deletion of either gene decreases sensitivity to drugs, and, conversely, over-expression of HXT11 increases sensitivity, leading to the suggestion that these proteins are not involved in drug efflux per se, but rather in modulating membrane permeability in cells which are over-expressing drug efflux $A B C$ transporters (Nourani et al., 1997).

Thirteen genes encoding toxicant efflux proteins, predominantly $A B C$ transporters and MF proteins, are present on this M. graminicola microarray. The lack of transporters identified as azole-responsive in this study is therefore somewhat surprising. However, previous microarray studies of the transcriptional response of S. cerevisiae (Bammert and Fostel, 2000) and C. albicans (De Backer et al., 2001) to azoles have also failed to detect significant up-regulation of efflux proteins. This is in contrast to earlier candidate gene approaches [e.g. the identification of CDR1 induction in response to fluconazole in C. albicans (Hernaez et al., 1998)]. Kontoyiannis and May (2001) suggested conditions of microarray studies, such as azole concentration and exposure time, were not optimal for detection of transporter gene expression. This might also apply to the current study, as only a single time point at a single fungicide concentration has been studied in detail. However, if a transporter is directly involved in exporting epoxiconazole out of the M. graminiola cell, it may simply be that it is not represented on this microarray. Up-regulation of hexose transporter $(\mathrm{mg}[0271])$ in response to azole treatment in isolates with greatest reductions in azole sensitivity provides evidence for this, as studies have suggested that homologues of $\mathrm{mg}[0271]$ are involved in regulating membrane permeability in $S$. cerevisiae cells overexpressing efflux pumps (Nourani et al., 1997).

\section{Response of other genes}

Azole resistance, for example in C. albicans, has been shown in both candidate gene (White, 1997) and microarray (Rogers and Barker, 2003) studies to be conferred, at least in part, by the constitutive over-expression of genes in resistant isolates. In this study, the expression of all genes identified as constitutively upregulated in less sensitive isolate $\mathrm{G} 303$, for example $\mathrm{mg}$ [0194] and mgc03e06f, decreased upon epoxiconazole treatment. It seems unlikely therefore that these genes play a role in reducing azole sensitivity in $\mathrm{G} 303$, although they may confer an improved capacity of this isolate to compete, infect and reproduce compared with the sensitive isolate IP0323. For example, $\mathrm{mg}$ [0194] is a homologue of the yeast stress response protein rds1 (Ludin et al., 1995). This gene has been shown to be highly expressed in pycnidiospores and late-stage in planta infection in $M$. graminicola isolate IP0323, but not in vitro, suggesting a specific role for this gene in reproduction and infection (Keon et al., 2005a,b). EST mgc03e06f encodes a homologue of the antibiotic response protein CipA of $A$. nidulans. CipA expression in A. nidulans is responsive to concanamycin A produced by Streptomyces (Melin et al., 2002). Therefore, the product of mgc03e06f may also afford $M$. graminicola protection against antibiotics produced by antagonistic microorganisms in the wheat phylloplane.

Microarray analyses identified one unclassified gene, mgc03g08f, as azole induced in G303. None were detected in studies of IP0323. This gene is annotated as a homologue of a $C$. neoformans mating-type locus marker, FAO1 (Lengeler et al., 2002), and has high homology to an iron/ascorbate oxidoreductase from A. fumigatus. Very high transcript levels of this gene (greater than 50 -fold increase) were also detected in the less sensitive isolate R6-55 after epoxiconazole treatment. No members of this protein family have previously been associated with response to azole in studies of fungal pathogens. In plants, these proteins have been suggested to play a role in detoxifying ROS (Qiu et al., 2004). Consistent with an effect of epoxiconazole on components of the mitochondrial respiratory chain, induction of mgc $03 \mathrm{~g} 08 \mathrm{f}$ may be in response to changing the oxidative state of the cell. Further functional studies are required to determine the exact impact of the product of this gene on azole sensitivity.

\section{CONCLUSIONS}

Profiling the response of $M$. graminicola to epoxiconazole using a CDNA microarray representing around one-quarter of the genome has confirmed ergosterol biosynthesis as the primary target of this compound and, furthermore, demonstrated an additional effect on components of the mitochondrial respiratory 
chain. The findings presented here are, in general, consistent with previous studies of the response of fungi to azoles. The reported anomalies, for example down-regulation of a putative erg6 gene after azole treatment, are most probably a consequence of misannotation of the EST set. The forthcoming release of the M. graminicola annotated genome sequence should resolve these inconsistencies. Comparisons of constitutive and azole-induced expression profiles between an azole-sensitive and less sensitive isolate failed to identify a gene, for example a drug efflux protein, directly responsible for the reduced azole sensitivity phenotype. However, a gene encoding a hexose transporter has been shown to be more highly expressed in least sensitive isolates. Although probably not directly involved in azole efflux, this protein may modulate the membrane activity of isolates over-expressing an, as yet unidentified, efflux protein. In the future, studies using whole genome microarrays will facilitate the identification of genes, and therefore mechanisms (other than target site alteration) responsible for decreased azole sensitivity in $M$. graminicola.

\section{EXPERIMENTAL PROCEDURES}

\section{Fungal isolates}

M. graminicola isolates G303, R6-31, R6-32, R6-40 and R6-55 (Table 1) were obtained following the protocol described in Fraaije et al. (2007). Briefly, wheat leaves with lesions were stapled on to sterile moist filter paper, placed in up-turned Petri dishes and incubated overnight at $23^{\circ} \mathrm{C}$. Single cirri, emerging from pycnidia, were collected using sterile forceps and suspended in $30 \mu \mathrm{L}$ of sterile distilled water. Suspensions were streaked on to yeast potato dextrose agar (YPD; ForMedium, Norwich, UK) amended with penicillin $\mathrm{G}$ sodium and streptomycin sulphate at $100 \mu \mathrm{g} / \mathrm{mL}$ and incubated for 5 days at $23^{\circ} \mathrm{C}$. Single colonies grew in the form of yeast-like budding cells. Single spore isolates were propagated by transferring single colonies to fresh YPD plates. Isolates were stored in $80 \%$ glycerol at $-80^{\circ} \mathrm{C}$ until epoxiconazole sensitivity testing.

\section{Epoxiconazole sensitivity testing}

Sensitivity assays were also according to Fraaije et al. (2007), with a few modifications. One hundred microlitres of $2 \times$ potato dextrose broth (PDB; Sigma-Aldrich, Inc., St. Louis, MO) amended with decreasing concentrations of epoxiconazole $(30,10,3.3$, $1.1,0.37,0.123,0.041,0.014,0.005,0.0015$ and $0.0005 \mathrm{mg} / \mathrm{L}$ ) was added to wells of flat-bottomed microtitre plates (TPP 92696 test plates, Trasadingen, Switzerland). After 7 days' growth at $15^{\circ} \mathrm{C}$ on YPD to ensure yeast-like growth, isolates were suspended in $5 \mathrm{~mL}$ sterile distilled water. Aliquots of $100 \mu \mathrm{L}$ of isolate spore suspensions $\left(1 \times 10^{5}\right.$ spores $\left./ \mathrm{mL}\right)$ were added to each well. Plates were incubated for 4 days at $23^{\circ} \mathrm{C}$, and growth measured by absorbance at $630 \mathrm{~nm}$ using an MRX plate reader (Dynex Tech- nologies, Chantilly, VA). Fungicide sensitivities were determined as $50 \%$ effective concentration $\left(\mathrm{EC}_{50}\right)$ using a dose-response relationship (Table 1).

\section{CYP51 sequencing}

CYP51 genes of M. gramincola isolates IP0323, G303, R6-31, R6-32, R6-40 and R6-55 were amplified and sequenced using the primers, PCR conditions and protocols outlined in Cools et al. (2005a). Amplifications were carried out using Phusion highfidelity DNA polymerase to ensure the validity of sequences (Finnzymes, Espoo, Finland).

\section{Growth of fungal cultures for microarray and quantitative RT-PCR analyses}

M. graminicola isolate spore suspensions were added to $100 \mathrm{~mL}$ of PDB (Sigma) at a final concentration of $4 \times 10^{5} \mathrm{spores} / \mathrm{mL}$. Cultures were grown at $23{ }^{\circ} \mathrm{C}$ with shaking ( 220 r.p.m.). After $48 \mathrm{~h}$, with isolates in the linear phase of growth (data not shown), epoxiconazole was added to each culture at $2 \mathrm{mg} / \mathrm{L}$, a concentration lethal to isolate IPO323 and around 50\% effective $\left(\mathrm{EC}_{50}\right)$ for isolate G303. Isolate IP0323 has previously been treated at the approximate $\mathrm{EC}_{50}(0.1 \mathrm{mg} / \mathrm{L})$ with no effect on expression profile (data not shown). This finding is in agreement with those of Jia et al. (2000). This treatment was therefore not continued. Tissue was harvested by filtration after either 1 or $24 \mathrm{~h}$ further growth at $23^{\circ} \mathrm{C}$ with shaking (220 r.p.m.). Filtered tissue was snap frozen in liquid nitrogen and stored at $-80^{\circ} \mathrm{C}$.

\section{RNA extraction and microarray hybridization and scanning}

Protocols for RNA extraction and microarray hybridization were essentially those described by Keon et al. (2005b) with some modification. Total RNA was extracted from freeze-dried M. graminicola tissue with TRIZOL reagent (Invitrogen, Carlsbad, CA) following the manufacturer's protocol. A subsequent overnight incubation of extracts in $4 \mathrm{~m}$ lithium chloride was used to purify RNA further. Labelling of isolated total RNA with reactive Alexafluor dyes was carried out using the Superscript Indirect CDNA Labelling System (Invitrogen) following the recommended protocol. Twenty micrograms of total RNA was used in each labelling reaction. Samples were labelled with either Alexafluor 555 (equivalent to Сy3) detected in the green channel, or Alexaflour 647 (equivalent to Cy5) detected in the red channel. Hybridization and washing steps were as outlined at the COGEME website (http://www.cogeme.man.ac.uk). Details of the M. graminicola microarray, including quality control experiments previously carried out, are described by Keon et al. (2005a,b). The annotated unigene set represented on the microarray is also available at 
Table 5 Primers used for quantitative RT-PCR studies.

\begin{tabular}{lll}
\hline & Sequence $\left(5^{\prime}-3^{\prime}\right)$ & \\
\cline { 2 - 3 } Target & Forward & Reverse \\
\hline $\begin{array}{ll}\text { 3-tubulin } \\
\text { CYP51/erg11 }\end{array}$ & AGAGAGCCTCGTTGTCAATGC & CGGTATGGGAACACTTCTCATCAG \\
mga1012f & ACACGAGCCCCATGGACGA & CGCCGATACATCTGCGTCGT \\
mg[0194] & CGCTGTCGATCAACGCTGTCTAT & CTTGGTGTTCGACTTGCTCTCCTC \\
mga0888f & ACCTCGCCTGGATCGCAAAG & TGGAGGGGGGACCAGTGACAGAGT \\
mgc19a12f & CGCCCGGCAGTACGAACTCTA & TTGTTCCAGGCGTAAGTGGCTAG \\
mg[0271] & TGCTCTGTGCACGTTTGCCTT & CATTACGCTCAGCCTTCTCCGA \\
mgC03g08f & ACGAACTTTGGCCTCAGTCAGGA & GTTCGGGAAGGAATTTGAAGACGT \\
\hline
\end{tabular}

the Phytopathogenic Fungi and Oomycete EST Database (http:// cogeme.ex.ac.uk/). Microarray slides were scanned using an Axon 4000B (Axon Instruments, Foster City, CA) scanner with a spot size of $5 \mu \mathrm{m}$. Preliminary analysis of the data output using GenePix software (Axon Instruments) was used to optimize scanning parameters.

\section{Microarray data analysis}

Output data were analysed using the methods described by Keon et al. (2005b) with the exception that eight replicates (four replicates of two repeat experiments) of GenePix results files were analysed to determine constitutive gene expression differences between IP0323 and G303, and to compare expression data after $24 \mathrm{~h}$ of epoxiconazole exposure to untreated samples for both IP0323 and G303. Microarray data were normalized by per spot and per chip intensity-dependent (Lowess) normalization. Up- and down-regulated transcripts were determined by filtering on confidence at a $t$-test $P$ value of 0.01 using the Benjamini and Hochberg multiple testing correction. Only genes with transcript levels differing two-fold or more were considered as differentially expressed. Blastx searches against the NCBI GenBank fungal genome database were used to verify annotations of differentially expressed genes.

\section{Hierarchical clustering}

Ratios of fluorescence (green/red) signals from eight replicate experiments after $24 \mathrm{~h}$ of epoxiconazole exposure, and four replicate experiments after $1 \mathrm{~h}$ of exposure (IP0323 treated/IP0323 untreated, and G303 treated/G303 untreated) were subtracted from fluorescent data generated for untreated IP0323. Data were logged to base two and stored in tab-delimited form. Cluster 3.0 (Eisen et al., 1998) was used to cluster data hierarchically using a Euclidean similarity matrix and the average-linkage clustering algorithm. TreeView 1.0.13 was used to view the output files from the clustering process. Blastx searches against the NCBI GenBank fungal genome database were used to verify annotations of genes emerging from cluster analyses.

\section{Quantitative RT-PCR analysis}

Five micrograms of total RNA was reversed transcribed with oligo(dT) ${ }_{20}$ using the SuperScript III First Strand Synthesis System (Invitrogen) according to the supplier's instructions. Quantitative RT-PCR reactions were carried using the SYBR Green Jumpstart Taq Ready Mix for Quantitative PCR (Sigma). cDNAs were diluted $(1: 10)$ and $5 \mu \mathrm{L}$ used in a $25-\mu \mathrm{L}$ reaction together with $0.25 \mu \mathrm{M}$ of each primer (Table 5). Thermal cycling conditions were as per the manufacturer's recommendations, with an annealing temperature of $56{ }^{\circ} \mathrm{C}$. Reactions were carried out on the $A B I 7500$ Real Time PCR System (Applied Biosystems) and data analysed using the 7500 SDS software (version 1.2.1, Applied Biosystems). All reactions were carried out in triplicate. Relative transcript abundances were calculated using the $2_{-}[\Delta][\Delta] \mathrm{Ct}$ method (Muller et al., 2002) with beta-tubulin as the endogenous control and untreated IP0323 as the calibrator sample. Data shown are the mean relative transcript abundances of two replicate experiments with standard deviations.

\section{ACKNOWLEDGEMENTS}

We would like to thank John Keon for technical assistance and Jason Rudd for his critical reading of the manuscript. This work was supported by the COGEME users fund (BB/C003462/1). Rothamsted Research receives grant aided support from the Biotechnology and Biological Sciences Research Council (BBSRC) of the UK.

\section{REFERENCES}

Agarwal, A.K., Rogers, P.D., Baerson, S.R., Jacob, M.R., Barker, K.S., Cleary, J.D., Walker, L.A., Nagle, D.G. and Clark, A.M. (2003) Genome-wide expression profiling of the response to polyene, pyrimidine, 
azole, and echinocandin antifungal agents in Saccharomyces cerevisiae. J. Biol. Chem. 278, 34998-35015.

Bammert, G.F. and Fostel, J.M. (2000) Genome-wide expression patterns in Saccharomyces cerevisiae: comparison of drug treatments and genetic alterations affecting biosynthesis of ergosterol. Antimicrob. Agents Chemother. 44, 1255-1265.

Boshoff, H.I.M., Myers, T.G., Copp, B.R., McNeil, M.R., Wilson, M.A. and Barry, C.E. III (2004) The transcriptional responses of Mycobacterium tuberculosis to inhibitors of metabolism. Novel insights into drug mechanisms of action. J. Biol. Chem. 279, 40174-40184.

Brun, S., Bergeìs, T., Poupard, P., Vauzelle-Moreau, C., Renier, G., Chabasse, D. and Bouchara, J.-P. (2004) Mechanisms of azole resistance in petite mutants of Candida glabrata. Antimicrob. Agents Chemother. $48,1788-1796$.

Clark, W.S. (2006) Septoria tritici and azole performance. Fungicide resistance: are we winning the battle but losing the war? Aspects Appl. Biol. 78, 127-132.

Cools, H.J., Fraaije, B.A., Kim, S.H. and Lucas, J.A. (2006) Impact of changes in the target P450 CYP51 enzyme associated with altered triazole-senstivity in fungal pathogens of cereal crops. Biochem. Soc. Trans. 34, 1219-1222.

Cools, H.J., Fraaije, B.A. and Lucas, J.A. (2005a) Molecular examination of Septoria tritici isolates with reduced sensitivities to triazoles. In: Modern Fungicides and Antifungal Compounds IV (Dehne, H.W., Gisi, U., Kuck, K.-H., Russell, P.E. and Lyr, H., eds), pp. 103-114. Alton, UK: BCPC.

Cools, H.J., Fraaije, B.A. and Lucas, J.A. (2005b) Molecular mechanisms correlated with changes in triazole sensitivity in isolates of Mycosphaerella graminicola. In: Proceedings of the BCPC Congress, Crop Science and Technology 2005, 1, pp. 267-274. Alton, UK: BCPC.

Daum, G., Lees, N.D., Bard, M. and Dickson, R. (1998) Biochemistry, and cell biology of lipids of Saccharomyces cerevisiae. Yeast, 14, 1471-1510.

De Backer, M.D., Ilyina, T., Ma, X.-J., Vandoninck, S., Luyten, W.H.M.L. and Bossche, H.V. (2001) Genomic profiling of the response of Candida albicans to itraconazole treatment using a DNA microarray. Antimicrob. Agents Chemother. 45, 1660-1670.

De Waard, M.A., Andrade, A.C., Hayashi, K., Schoonbeek, H.-J., Stergiopoulos, I. and Zwiers, L.-H. (2006) Impact of fungal drug transporters on fungicide sensitivity, multidrug resistance and virulence. Pest Manag. Sci. 62, 195-207.

Delye, C., Bousset, L. and Corio-Costet, M.F. (1998) PCR cloning and detection of point mutations in the eburicol 14a-demethylase (CYP51) gene from Erysiphe graminis f. sp. hordei, a 'recalcitrant' fungus. Curr. Genet. 34, 399-403.

Delye, C., Laigret, F. and Corio-Costet, M.F. (1997) A mutation in the sterol 14a-demethylase gene in Uncinular necator that correlates with resistance to sterol biosynthesis inhibitors. Appl. Environ. Microbiol. 63, 2966-2970.

Eisen, M.B., Spellman, P.T., Brown, P.O. and Botstein, D. (1998) Cluster analysis and display of genome-wide expression patterns. Proc. Natl Acad. Sci. USA, 95, 14863-14868.

Ferreira, M.E.D.S., Malavazi, I., Savoldi, M., Brakhage, A.A., Goldman, M.H.S., Kim, H.S., Nierman, W.C. and Goldman, G.H. (2006) Transcriptome analysis of Aspergillus fumigatus exposed to voriconazole. Curr. Genet. 50, 32-44.

Fraaije, B.A., Cools, H.J., Fountaine, J., Lovell, D.J., Motteram, J., West, J.S. and Lucas, J.A. (2005) The role of ascospores in further spread of Qol-resistant cytochrome b alleles (G143A) in field populations of Mycosphaerella graminicola. Phytopathology, 95, 933-941.
Fraaije, B.A., Cools, H.J., Kim, S.-H., Motteram, J., Clark, W.S. and Lucas, J.A. (2007) A novel substitution I381V in the sterol 14a-demethylase (CYP51) of Mycosphaerella graminicola is differentially selected by azole fungicides. Mol. Plant Pathol, 8, 245-254.

Fraaije, B.A., Lucas, J.A., Clark, W.S. and Burnett, F.J. (2003) Qol resistance development in populations of cereal pathogens in the UK. In: Proceedings of the BCPC Congress, Crop Science and Technology 2005, 2. Alton, UK: BCPC, pp. 689-694.

Hamamoto, H., Hasegawa, K., Nakaune, R., Lee, J.L., Makizumi, Y., Akutsu, K. and Hibi, T. (2000) Tandem repeat of a transcriptional enhancer upstream of the sterol 14a-demethylase gene (CYP51) in Penicillium digitatum. Appl. Environ. Microbiol. 66, 3421-3426.

Hardwick, N.V., Jones, D.R. and Royle, D.J. (2001) Factors affecting diseases of winter wheat in England and Wales, 1989-1998. Plant Pathol. $50,453-462$

Hernaez, M.L., Gil, C., Pla, J. and Nombela, C. (1998) Induced expression of the Candia albicans multidrug resistance gene CDR1 in response to fluconazole and other antifungals. Yeast, 14, 517-526.

Jia, M.H., Larossa, R.A., Lee, J.-M., Rafalski, A., DeRose, E., Gonje, G. and Xue, Z. (2000) Global expression profiling of yeast treated with an inhibitor of amino acid biosynthesis, sulfometuron methyl. Physiol. Genom. 3, 83-82.

Kelly, S.L., Lamb, D.C. and Kelly, D.E. (1999) Y132H substitution in Candida albicans sterol 14a-demethylase confers fluconazole resistance by preventing binding to haem. FEMS Microbiol. Lett. 180, 171-175.

Kenna, S., Bligh, H.F.J., Watson, P.F. and Kelly, S.L. (1989) Genetic and physiological analysis of azole sensitivity in Saccharomyces cerevisiae. J. Med. Vet. Mycol. 27, 397-406.

Keon, J., Rudd, J.J., Antoniw, J., Skinner, W., Hargreaves, J. and Hammond-Kosack, K. (2005a) Analysis of expressed sequence tags from the wheat leaf blotch pathogen Mycosphaerella graminicola (anamorph Septoria tritici). Fungal Genet. Biol. 42, 376-389.

Keon, J., Rudd, J.J., Antoniw, J., Skinner, W., Hargreaves, J. and Hammond-Kosack, K. (2005b) Metabolic and stress adaptation by Mycosphaerella graminicola during sporulation in its host revealed through microarray transcription profiling. Mol. Plant. Pathol. 6, 527-540.

Kontoyiannis, D.P. and May, G.S. (2001) Identification of azole-responsive genes by microarray technology: why are we missing all the efflux transporter genes? Antimicrob. Agents Chemother. 45, 3674-3676.

Lamb, D.C., Kelly, D.E., White, T.C. and Kelly, S.L. (2000) The R467K amino acid substitution in Candida albicans sterol 14a-demethylase causes drug resistance through reduced affinity. Antimicrob. Agents Chemother. 44, 63-67.

Lengeler, K.B., Fox, D.S., Fraser, J.A., Allen, A., Forrester, K., Dietrich, F.S. and Heitman, J. (2002) Mating-type locus of Cryptococcus neoformans: a step in the evolution of sex chromosomes. Eukaryot. Cell, 1, 704-718.

Liu, T.T., Lee, R.E.B., Barker, K.S., Lee, R.E., Wei, L., Homayouni, R. and Rogers, P.D. (2005) Genome-wide expression profiling of the response to azole, polyene, echinocandin, and pyrimidine antifungal agents in Candida albicans. Antimicrob. Agents Chemother. 49, 22262236.

Ludin, K.M., Hilti, N. and Schweingruber, M.E. (1995) Schizosaccharomycespombe rds1, an adenine-repressible gene regulated by glucose, ammonium, phosphate, carbon-dioxide and temperature. Mol. Gen. Genet. 248, 439-445.

Melin, P., Schnurer, J. and Wagner, E.G. (2002) Proteome analysis of Aspergillus nidulans reveals proteins associated with the response to the 
antibiotic concanamycin A, produced by Streptomyces species. Mol. Genet. Genomics, 267, 695-702.

Muller, P.Y., Janovjak, H., Miserez, A.R. and Dobbie, Z. (2002) Processing of gene expression data generated by quantitative real-time RT-PCR. Biotechniques, 32, 1372-1379.

Nakaune, R., Adachi, K., Nawata, O., Tomiyama, M., Akutsu, K. and Hibi, H. (1998) A novel ATP-binding cassette transporter involved in multidrug resistance in the phytopathogenic fungus Penicillium digitatum. Appl. Environ. Microbiol. 64, 3983-3988.

Nourani, A., Wesolowski-Louvel, M., Delaveau, T., Jacq, C. and Delahodde, A. (1997) Multiple-drug-resistance phenomenon in the yeast Saccharomyces cerevisiae: involvement of two hexose transporters. Mol. Cell. Biol. 17, 5453-5460.

Perea, S., Lopez-Ribot, J.L., Kirkpatrick, W.R., McAtee, R.K., Santillan, R.A., Martinez, M., Calabrese, D., Sanglard, D. and Patterson, T.F. (2001) Prevalence of molecular mechanisms of resistance to azole antifungal agents in Candida albicans strains displaying high-level fluconazole resistance isolated from human immunodeficiency virus-infected patients. Antimicrob. Agents Chemother. 45, 2676-2684.

Qiu, X., Guan, P., Wang, M.-L., Moore, P.H., Zhu, Y.J., Hu, J., Borth, W. and Albert, H.H. (2004) Identification and expression analysis of BTH induced genes in papaya. Physiol. Mol. Plant Pathol. 65, 21-30.

Rogers, P.D. and Barker, K.S. (2003) Genome-wide expression profile analysis reveals coordinately regulated genes associated with stepwise acquisition of azole resistance in Candida albicans clinical isolates. Antimicrob. Agents Chemother. 47, 1220-1227.

Sanglard, D., Ischer, F. and Bille, J. (2001) Role of ATP binding-cassette transporter genes in high-frequency acquisition of resistance to azole antifungals in Candida glabrata. Antimicrob. Agents Chemother. 45, 1174-1183.
Sanglard, D., Ischer, F., Koymans, L. and Billie, J. (1998) Amino acid substitutions in the cytochrome P450 lanosterol 14a-demethylase (CYP51A1) from azole-resistant Candida albicans clinical isolates contribute to resistance to antifungal agents. Antimicrob. Agents Chemother. 42, 241-253.

Schnabel, G. and Jones, A.L. (2001) The 14a-demethylase (CYP51A1) gene is overexpressed in Venturia inaequalis strains resistant to myclobutanil. Phytopathology, 91, 102-110.

Shigematsu, M.L., Uno, J. and Arai, T. (1982) Effect of ketoconazole on isolated mitocondria from Candida albicans. Antimicrob. Agents Chemother. 21, 919-924.

Stergiopoulos, I., Van Nistelrooy, J.G.M., Kema, G.H.J. and De Waard, M.A. (2003) Multiple mechanisms account for variation in base-line sensitivity to azole fungicides in field isolates of Mycosphaerella graminicola. Pest Manag. Sci. 59, 1333-1343.

Vera, J.C., Castillo, G.R. and Rosen, O.M. (1991) A possible role for a mammalian facilitative hexose transporter in the development of resistance to drugs. Mol. Cell. Biol. 11, 3407-3418.

White, T.C. (1997) Increased mRNA levels of ERG16, CDR and MDR1 correlate with increases in azole resistance in Candida albicans isolates from a patient infected with human immunodeficiency virus. Antimicrob. Agents Chemother. 41, 1482-1487.

Wyand, R.A. and Brown, J.K.M. (2005) Sequence variation in the CYP51 gene of Blumeria graminis associated with resistance to sterol demethylase inhibiting fungicides. Fungal Genet. Biol. 42, 726735.

Zwiers, L.-H., Stergiopoulos, I., Van Nistelrooy, J.G.M. and De Waard, M.A. (2002) ABC transporters and azole sensitivity in laboratory strains of the wheat pathogen Mycosphaerella graminicola. Antimicrob. Agents Chemother. 46, 3900-3906. 